\title{
Apical membrane rupture and backward bile flooding in acetaminophen-induced hepatocyte necrosis
}

\author{
F-C Li', G-T Huang ${ }^{2}$, C-J Lin ${ }^{1}$, S-S Wang ${ }^{1}$, T-L Sun ${ }^{1}$, S-Y Lo ${ }^{1}$, W Lo ${ }^{1}$, L-L Chiou ${ }^{3}$, C-Y Dong ${ }^{*, 1,4}$ and H-S Lee ${ }^{\star, 2,4,5,6}$
}

Morphological changes of hepatocyte death have so far only been described on cells in culture or in tissue sections. Using a high-resolution and high-magnification multiphoton microscopic system, we recorded in living mice serial changes of acetaminophen (APAP)-induced hepatocyte necrosis in relevance to metabolism of a fluorogenic bile solute. Initial changes of hepatocyte injury included basal membrane disruption and loss of mitochondrial membrane potential. An overwhelming event of rupture at adjacent apical membrane resulting in flooding of bile into these hepatocytes might ensue. Belbs formed on basal membrane and then dislodged into the sinusoid circulation. Transmission electron microscopy disclosed a necrotic hepatocyte depicting well the changes after apical membrane rupture and bile flooding. Administration of the antidote $\mathrm{N}$-acetylcysteine dramatically reduced the occurrence of apical membrane rupture. The present results demonstrated a hidden but critical step of apical membrane rupture leading to irreversible APAP-induced hepatocyte injury.

Cell Death and Disease (2011) 2, e183; doi:10.1038/cddis.2011.68; published online 21 July 2011

Subject Category: Experimental Medicine

Necrosis of cells, including hepatocytes, has been morphologically characterized by an outward rupture of the plasma membrane leading to a loss of cell constituents. ${ }^{1-4}$ Knowledge of cell necrosis was mostly based on investigations on static cells in tissue sections or cells monitored in a culture. Investigations using tissue sections may cause confusion in chronological sequences and easily miss rare or short-lived events. Time-lapse studies on cells in culture may overcome these drawbacks. However, they may also miss many key features because in vivo intercellular interactions or 3-dimensional complex structures with physiological significance are difficult to reproduce correctly in a Petri dish. Many methods already exist to image cell death. ${ }^{5}$ However, studies on cell death by intravital imaging are still lacking.

Multiphoton microscopy allows high-resolution imaging of cells deep in various organs of living animals. ${ }^{6}$ Such real-time imaging has been successful in tracking cancer ${ }^{7}$ or immune ${ }^{8}$ cells in mice, but it has never been applied to study cell death. ${ }^{5}$ To study cell death using this approach, the power of both resolution and magnification of the imaging system must be high enough to resolve a single cell in the tissues. For acquiring such imaging in a mouse liver, we developed a metallic liver window device to fix a liver that is affected by movement caused by heartbeats. ${ }^{9,10}$ We have shown that this approach was powerful enough to image a single hepatocyte clearly. ${ }^{10}$ By using this technique, we studied acetaminophen (APAP)-induced hepatocyte necrosis in vivo.

APAP overdose is the leading cause of acute liver failure in Western countries. ${ }^{11}$ A portion of APAP in the liver is metabolized by cytochrome P-450 (CYP) 2E1 to produce reactive $N$-acetyl-p-quinoneimine (NAPQI), which may be detoxified by preferential binding to glutathione. When NAPQI is produced to a large amount sufficient to deplete nearly all the hepatocyte glutathione, this toxic metabolite will bind cellular proteins ${ }^{12}$ and leads to mitochondrial dysfunction, depletion of adenosine triphosphate, and, ultimately, to hepatocellular death. ${ }^{13,14}$ Bleb formation on the hepatocyte surface is the most prominent ultrastructual change of APAP toxicity on isolated hepatocytes. ${ }^{15}$ Using scanning electron microscopy, the injured liver displayed features of congestion, extravasation of erythrocytes into the Disse space, dilatation of bile canaliculi, and a spongy appearance of necrotic hepatocytes. ${ }^{16}$ However, information regarding sequential morphological changes during in vivo hepatocyte necrosis is still lacking. Here we showed the in vivo morphological changes with an emphasis on the unexpected event of canalicular membrane rupture and the flooding of bile back into these hepatocytes. These events were accompanied by an abrupt drop of mitochondrial membrane potential and might indicate a possible point of no return in hepatocyte necrosis.

\footnotetext{
${ }^{1}$ Department of Physics, National Taiwan University, Taipei, Taiwan; ${ }^{2}$ Department of Internal Medicine, National Taiwan University Hospital and National Taiwan University College of Medicine, Taipei, Taiwan; ${ }^{3}$ Liver Disease Prevention and Treatment Research Foundation, Taipei, Taiwan; ${ }^{4}$ Molecular Imaging Center, National Taiwan University, Taipei, Taiwan; ${ }^{5}$ Institute of Biotechnology, National Taiwan University, Taipei, Taiwan and ${ }^{6}$ Agricultural Biotechnology Research Center, Academia Sinica, Taipei, Taiwan

${ }^{*}$ Corresponding authors: H-S Lee, Institute of Biotechnology, National Taiwan University, 4F, No. 81, Chang-Xing Street, Taipei 106, Taiwan. Tel: + 88 629 726 51089 ; Fax: + 88623366 6001; E-mail: benlee@ntu.edu.tw

or C-Y Dong, Department of Physics, National Taiwan University, 4F, No. 81, Chang-Xing Street, Taipei 106, Taiwan. Tel: + 886233665155 ; Fax: + 886233665244 ; E-mail: cydong @phys.ntu.edu.tw

Keywords: acetaminophen; hepatocyte necrosis; multiphoton microscopy; intravital imaging

Abbreviations: APAP, acetaminophen; CFDA, carboxyfluorescein diacetate; FITC, fluorescein isothiocyanate; NAC, N-acetylcysteine; RITC, rhodamine B isothiocyanate; R6G, rhodamine 6G; TEM, transmission electron microscopy; TMRM, tetramethylrhodamine methylester

Received 27.4.11; revised 22.6.11; accepted 23.6.11; Edited by M Federici
} 


\section{Results}

Integrity of sinusoidal and canalicular membrane in APAP-injured hepatocytes. At $24 \mathrm{~h}$ after an overdose of APAP, mice were intravenously injected with sets of fluorochromes before starting imaging. Intravenous injection of rhodamine B isothiocyanate (RITC)-dextran or fluorescein isothiocyanate (FITC)-dextran allows imaging of sinusoidal veins. Normal hepatocytes would not take up dextran unless their plasma membranes are compromised allowing permeation of dextran from circulation. Injection of carboxyfluorescein diacetate (CFDA) allows imaging of bile canaliculi during excretion of this solute. Non-fluorogenic CFDA will turn into fluorogenic carboxyfluorescein (CF) after hydroxylation in hepatocytes and then be excreted from the canalicular membrane into bile canaliculi. ${ }^{9}$ Thus, both labeling approaches applied together can simultaneously provide information on the integrity of both sinusoidal (basal) and canalicular (apical) membranes. At $50 \mathrm{~min}$ post-CFDA injection, several hepatocytes, either discrete or in clusters, were detected more intensely retaining green $\mathrm{CF}$ fluorescence (green arrows) than neighboring normallooking hepatocytes in the liver of an APAP-treated mouse (Figure 1a). Careful analysis of the real-time images revealed two types of CF-retaining cells. Most common were those that showed brighter green than normal-looking hepatocytes within 2-3 min post-CFDA injection, thus, referred to as 'early CF-retaining (ECFR) cells'. The others, which began to get brighter later, were referred to as 'new CF-retaining (NCFR) cells'. Traced back to zero time (after RITC-dextran injection but before CFDA injection), both types of CF-retaining cells were all pre-labeled by RITC-dextran, though retention tended to be less intense in ECFR cells. In contrast, many RITC-dextran pre-labeled cells metabolized CF normally as normal-looking hepatocytes during the observation. They were, thus, referred to as 'dextran-labeled but did not retain CF (DLCFNR) cells' (red arrows in Figure 1a). Very occasionally, NCFR cells were not pre-labeled by RITCdextran. In the normal mouse, these changes were rarely seen (Figure 1b). Only one ECFR cell was noted (green arrow) and this might indicate a physiologically rare turnover cell. Figure 1d shows the numbers of respective cell types in the $660 \times 660 \mu \mathrm{m}$ microscopic areas. Figure 1e shows representative kinetic curves of green CF fluorescence in normal, ECFR, NCFR, and DLCFNR cells. The kinetics in NCFR cells were characterized by a resurgence of the fluorescence within the course of observation. The intensities in ECFR cells were persistently higher than those in normal cells. Basically, the kinetics in DLCFNR cells is similar to normal cells. In NCFR cells, the green fluorescence signals tended to appear focally inside the cells (white arrows in
Figure 1c) and then spread out over the whole cell. These CF signals most likely came from bile canaliculi because, at that time, most CF molecules were excreted into and accumulated in the canaliculi.

Canalicular membrane rupture resulting in bile flooding in NCFR hepatocytes. To prove that the CF fluorescence signals that freshly appeared in the NCFR cells came from adjacent bile canaliculi, better examples were sought, which showed their spatial orientations. Two examples were most revealing. In example 1 (Figure $2 a$ and video 1 ), a striking event was noticed in which an RITC-dextran-labeled hepatocyte suddenly developed a ballooning change in a bile canaliculus, which soon ruptured and flooded bile back into this swollen hepatocyte. Variable-sized blebs that protruded into the sinusoids were visible as the cell became strongly stained by CF. Some blebs were dislodged and floated away into the sinusoids (blue arrows in Figure 2a). Example 2 (Figure $2 b$ and video 2 ) more clearly shows the ballooning changes of the canaliculi before rupturing into the neighboring hepatocytes.

ECFR cells were those which already experienced canalicular membrane rupture. On the basis of these observations, ECFR cells were hypothesized to be descendents of NCFR cells. To prove this, the observation period was extended by repeating the CFDA injection. In this imaging system, fluorogenic CF signals in bile canaliculi are only detectable between 3 and 60 min post-single injection of CFDA. ${ }^{10}$ To overcome this, CFDA was re-injected at $60 \mathrm{~min}$ and observed for another $60 \mathrm{~min}$. In this experiment, six compromised hepatocytes were recorded sequentially (Figure 3 and video 3). During the first course (Figure 3a), cells 1,2 , and 3 were lightened by CF at $35-46 \mathrm{~min}$. They were accordingly labeled as NCFR cells. Loss of their cell contents into sinusoids was also noted. Cell 1 was not seen in the second course because of a subtle $z$-axis shift. In the second course (Figure $3 b$ ), cells 2 and 3 apparently became ECFR cells, thus providing evidence that ECFR cells were those which previously suffered from canalicular membrane rupture.

Sytox Blue (m.w. $\sim 400$ daltons) was injected at the beginning of the second course. Here cells 4 and 5 are shown stained by Sytox but not by RITC-dextran at $1 \mathrm{~min}$ and $9 \mathrm{~min}$, respectively. These two cells developed abrupt swelling and bile flooding, suggesting that the canalicular membrane rupture occurred at $45 \mathrm{~min}$ and $27 \mathrm{~min}$, respectively. Strikingly, cell 6 also experienced canalicular membrane rupture at 45 min. However, it was not previously labeled by either RITCdextran or Sytox.

Figure 1 APAP-injured hepatocytes indicated by RITC-dextran and CF labeling. (a) In the liver of an APAP-treated mouse, several hepatocytes abnormally retained CF (green arrows) and several others were labeled red by RITC-dextran (red arrows) at 50 min post-CFDA injection. (b) In a normal liver, only one cell abnormally retained CF was noted (green arrow). (c) Serial images of the boxed area in a show focal emergence of green CF fluorescence inside two NCFR cells (white arrows). A green arrow indicates an ECFR cell and a red arrow indicates a DLCFNR cell. (d) Numbers of abnormal cells in the APAP-treated and normal control mouse. $N=$ DLCFNR cell. (e) Kinetics of CF fluorescence in a normal (1), NCFR (2), ECFR (3), and DLCFNR (4) cell, respectively, indicated in c, 40 min. Note the resurgence in the NCFR cell (arrow). Scale bars indicate $100 \mu \mathrm{m}$ 
a
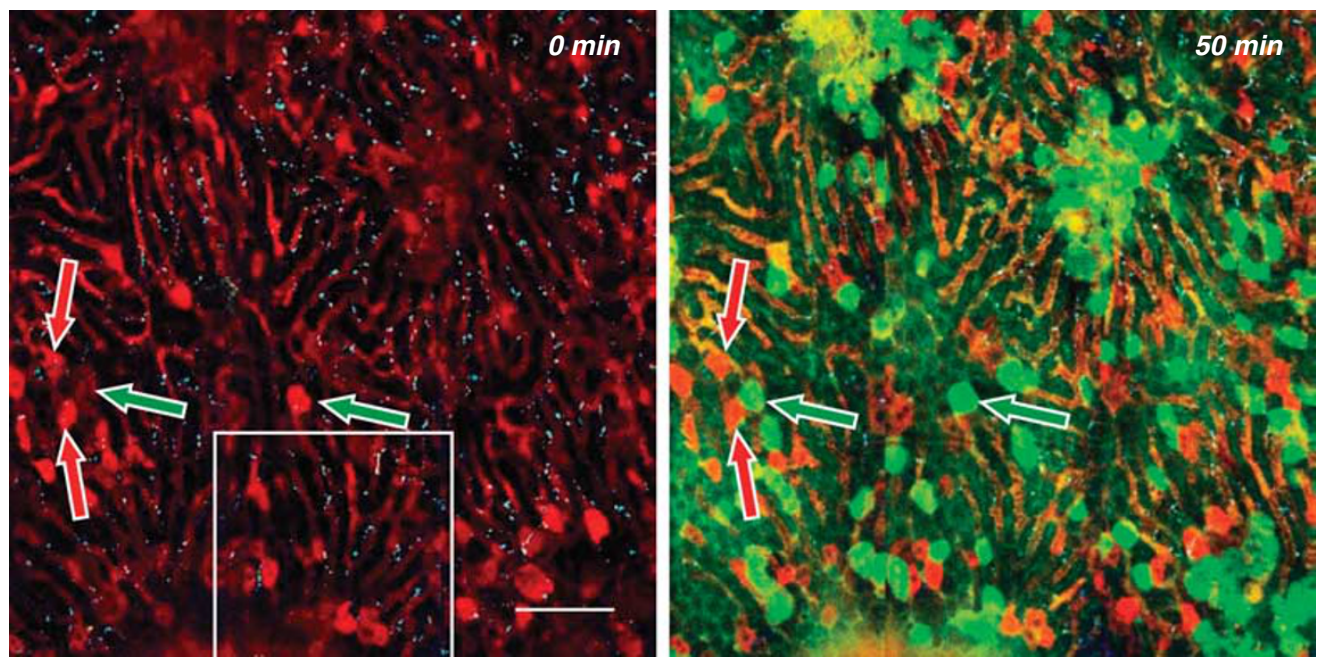

b
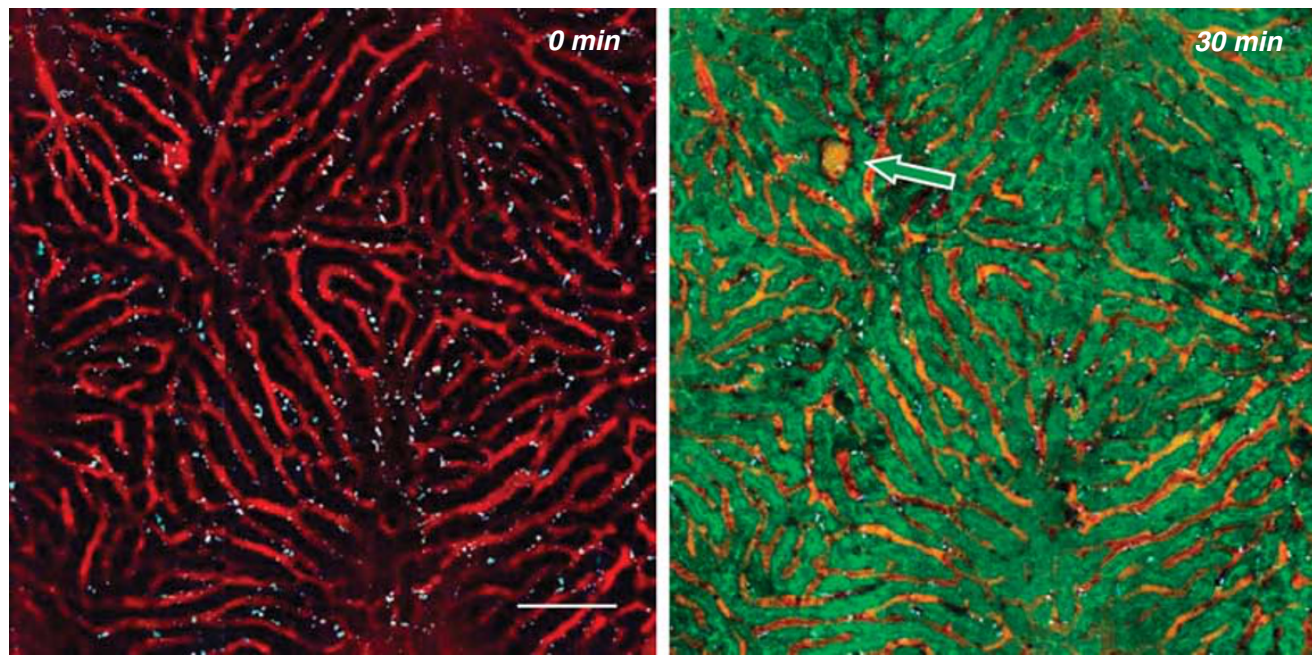

c
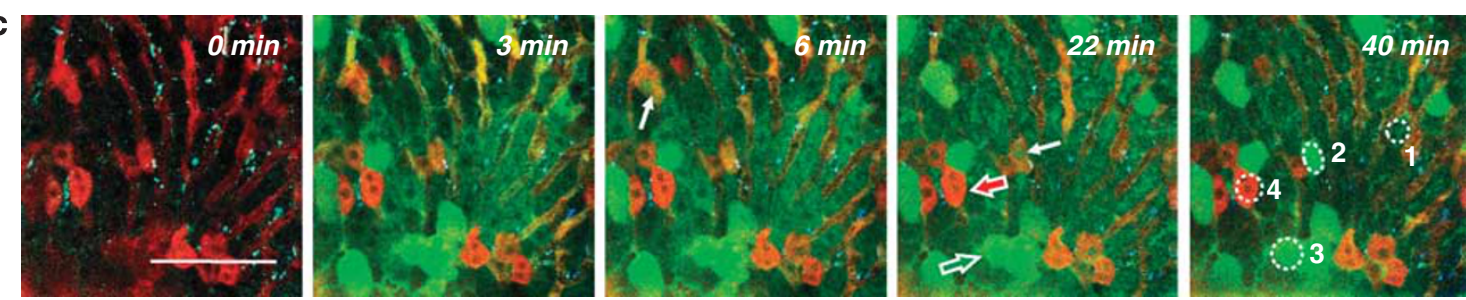

d

\begin{tabular}{cccccc} 
& \multicolumn{3}{c}{ Dextran (+) } & & Dextran (-) \\
\cline { 2 - 4 } \cline { 6 - 6 } & $\mathrm{N}$ & ECFR & NCFR & & NCFR \\
\hline APAP & 34 & $>90$ & 10 & & 1 \\
Normal & 0 & 1 & 0 & & 0 \\
\hline
\end{tabular}

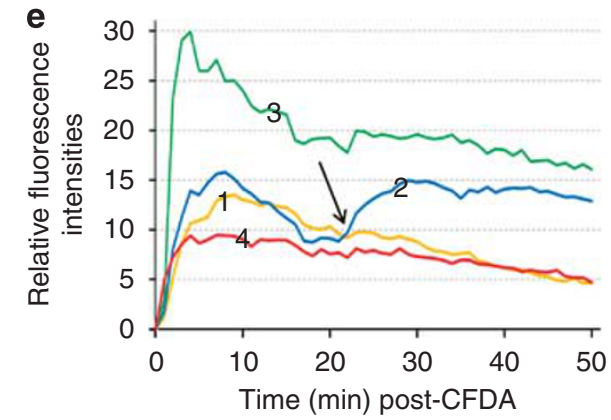



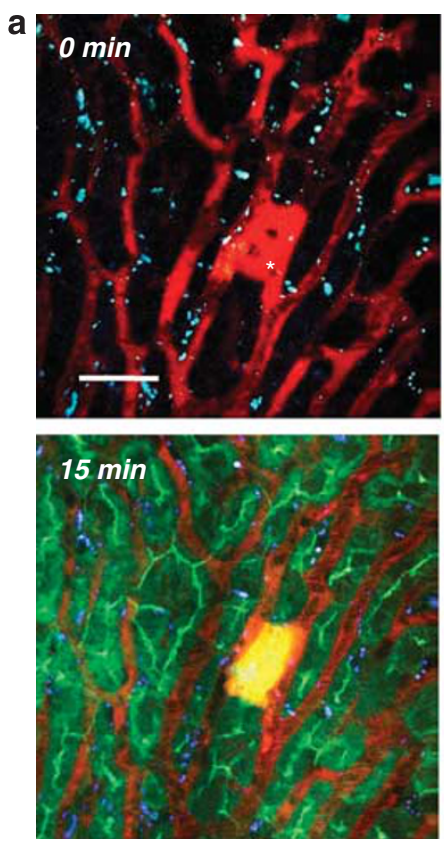
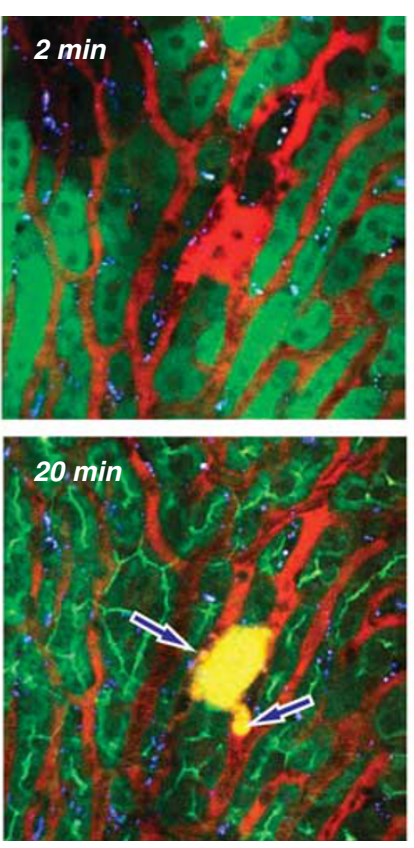
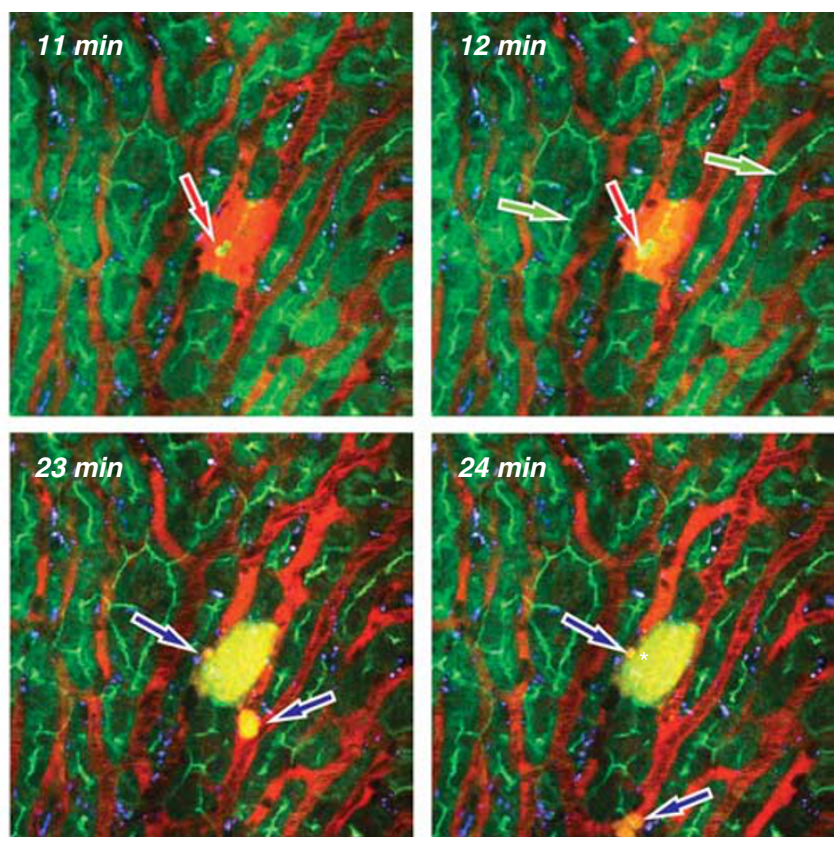
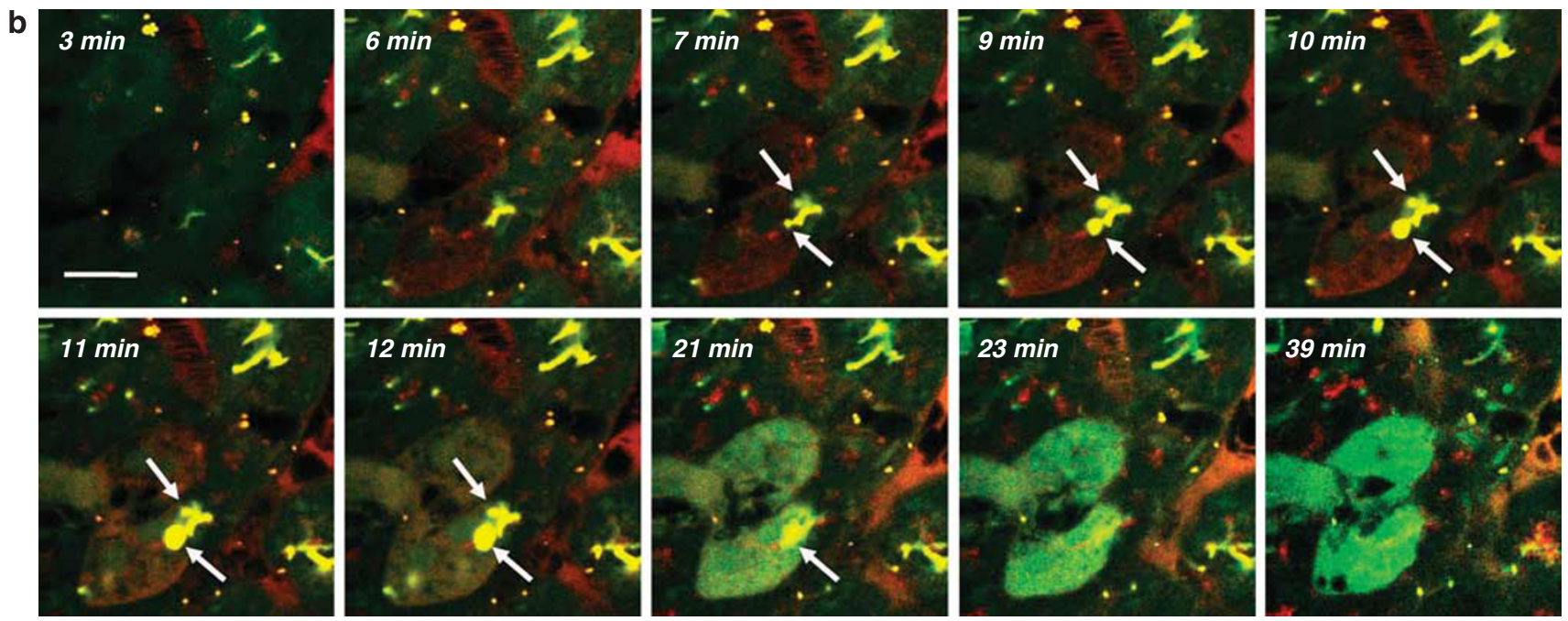

Figure 2 Evidence of canalicular membrane rupture in NCFR cells. (a) A hepatocyte pre-labeled by RITC-dextran (indicated by asterisk (*)) developed ballooning and rupturing of an overlying bile canaliculus. Red arrows indicate ballooning canaliculus, green arrows indicate bile canaliculi and blue arrows indicate blebs, which might be dislodged into sinusoids. (b) Another example more clearly shows progressive ballooning change of 2 bile canaliculi (arrows) that blew up later. Scale bars indicate $40 \mu \mathrm{m}$ in a and $20 \mu \mathrm{m}$ in $\mathbf{b}$

Are APAP-injured hepatocytes reversible? If basal membrane-disrupted cells are reversible, identifying them using the present labeling methods may be difficult because dextran or Sytox molecules may still be retained inside the cells after their recovery. Thus, a mitochondrial dye, rhodamine 6G (R6G), was used, which binds tightly to and accumulates in mitochondria. ${ }^{17}$ This dye has been used for intravital microscopic studies of white blood cells. ${ }^{18} \mathrm{~A}$ similar fluorochrome, tetramethylrhodamine methylester (TMRM), is taken up by polarized mitochondria but released as mitochondria become depolarized. Thus, TMRM became an indicator of mitochondrial membrane potential. ${ }^{19}$ First, R6G and FITC-dextran was injected intravenously. Figure 4 shows well staining of R6G on normal hepatocytes and white blood cells but not on the compromised hepatocytes that were permeated by FITC-dextran, suggesting that their sinusoidal membrane was already disrupted and that they might be either DLCFNR or CF-retaining cells. This characteristic indicates a tight correlation between mitochondrial dysfunction and sinusoidal membrane disruption. By injecting R6G, Sytox, and CFDA, three kinds of compromised hepatocytes were recorded. First, a hepatocyte stained by Sytox, but not R6G, later developed a canalicular membrane rupture (Figure 5a). This was typically a NCFR cell. CF fluorescence intensity in this cell resurged at around $20 \mathrm{~min}$ (arrow in Figure 5Da) coinciding with a canalicular membrane rupture and bile flooding. The intensity of R6G in this cell was persistently lower than that in 

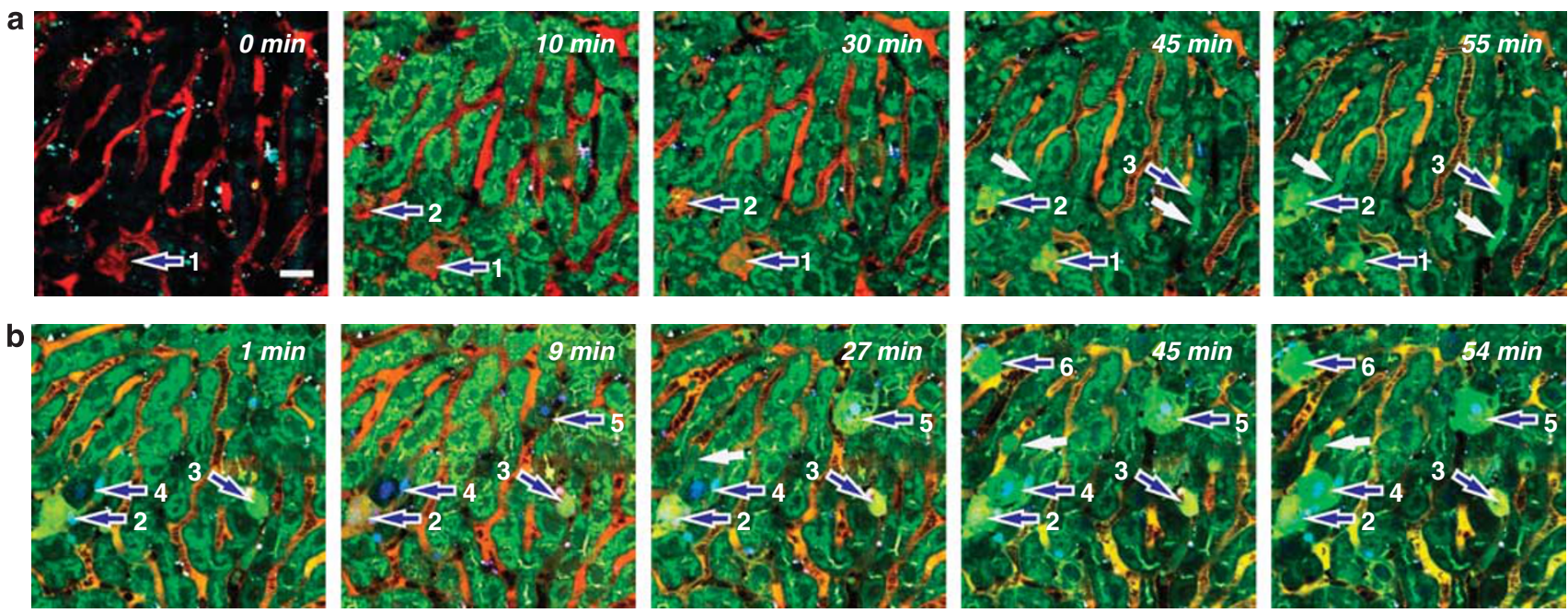

Figure 3 Sequential two courses (a, first b, second) of CFDA injections 60 min apart. Cells 1, 2, and 3 began to be greenly lightened up at 35-46 min with part of their cellular contents (white arrows) lost into sinusoids, suggesting they were NCFR cells in the first course. They became typical ECFR cells in the second course. Cells 4 and 5 initially labeled at nuclei by Sytox developed swelling and lightened up by CF at $45 \mathrm{~min}$ and $27 \mathrm{~min}$, respectively. Cell 6, without preceding labeling by either RITC-dextran or Sytox, was also abruptly lightened up at $45 \mathrm{~min}$. Blue arrows indicate numbered injured cells. Scale bar indicates $25 \mu \mathrm{m}$

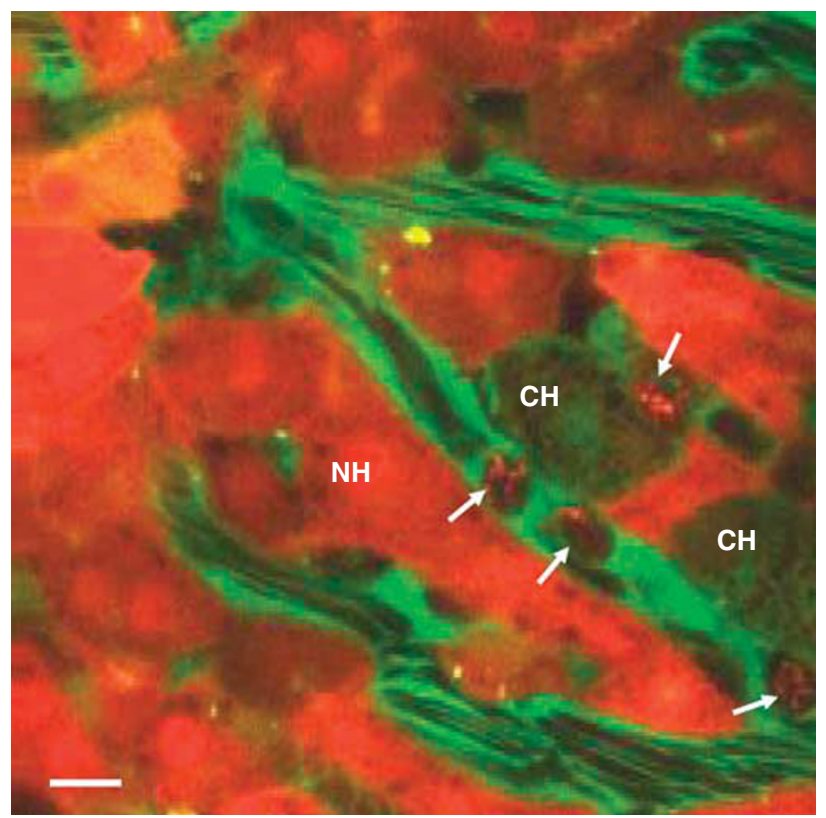

Figure 4 Co-existence of non-labeling by R6G and FITC-dextran permeation in APAP-injured hepatocytes. Normal hepatocytes $(\mathrm{NH})$ and white blood cells (indicated by arrows) were normally labeled by R6G, while compromised hepatocytes $(\mathrm{CH})$ were not. In contrast, the compromised hepatocytes were labeled by FITC-dextran. Scale bar indicates $10 \mu \mathrm{m}$

normal hepatocytes. Second, a cell well stained by R6G and lacking Sytox staining abruptly lightened at $37 \mathrm{~min}$ (Figure 5b). The kinetics of CF fluorescence also showed a resurgence at that time. More interestingly, R6G fluorescence in this cell abruptly dropped simultaneously with CF resurgence (arrow in Figure 5Db), strongly suggesting that $R 6 G$ is also a good indicator of mitochondrial dysfunction. Abrupt loss of R6G staining in this cell was, thus, most likely a result of bile acid-triggered mitochondrial depolarization. Massive flooding of bile into hepatocytes might bump their respiration system heavily enough to lead to irreversible cell death. Third, a cell initially stained by Sytox, but less R6G, soon recovered R6G staining, indicating the recovery of its vitality (Figure $5 \mathrm{c}$ ). The kinetics of R6G intensity in this cell showed a re-elevation to the normal level within $5 \mathrm{~min}$ (arrow in Figure 5Dc). The third cell suggested that mitochondriadepolarized hepatocytes and probably also basal membranedisrupted hepatocytes were still recoverable.

Hypothesis on the course of APAP-induced hepatocyte necrosis. A hypothesis regarding the outcomes of APAPinjured hepatocytes (Figure 6) was proposed. Sinusoidal membrane disruption coexisting with mitochondrial dysfunction is the initial injury caused by APAP. Hepatocyte injury in this stage may still be reversible. Canalicular membrane rupture with bile flooding into injured hepatocytes is a critical step toward irreversible cell injuries. This always, but not necessarily, occurs following a sinusoidal membrane defect or mitochondrial dysfunction. The cells post-canalicular membrane rupture made them become ECFR cells as bile solute CF may readily invade these dead cells via broken bile canaliculi.

Electron microscopic evidence supporting the hypothesis. To support this hypothesis, the APAP-injured liver was examined using transmission electron microscopy (TEM). This disclosed that a dead hepatocyte might well depict cell death after canalicular membrane rupture (Figure 7a). This cell became shrunken and its plasma had a ground-glass appearance. Bile lakes appeared with ragged margins, suggesting that a forceful tearing had occurred during its formation. These lakes were immediately opposed to the rough border of a bile canaliculus, suggesting that this forceful tearing was a result of inward flooding of bile from the 


\section{A}
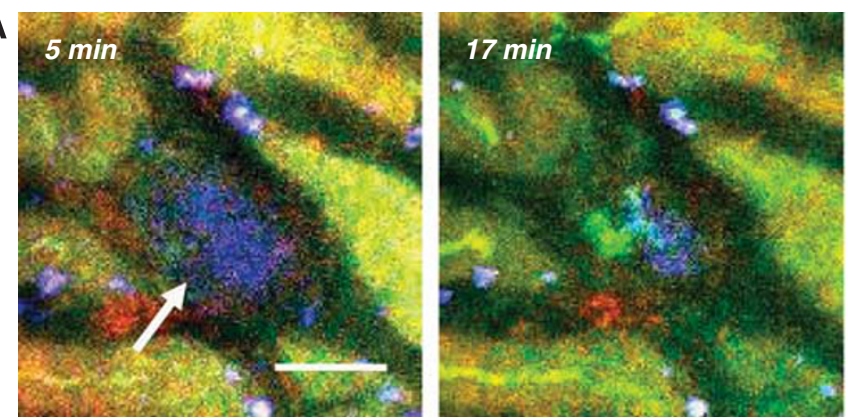

B

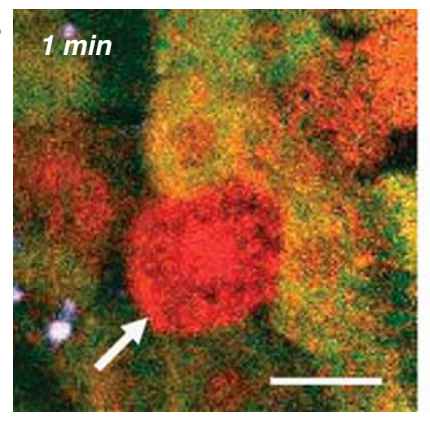

C

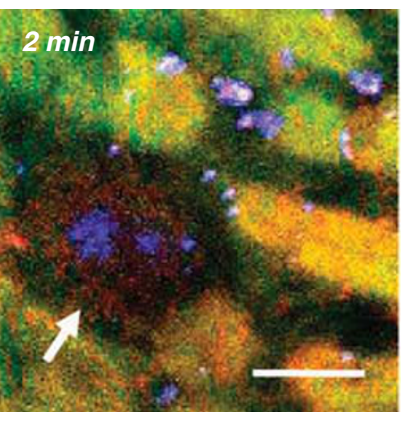

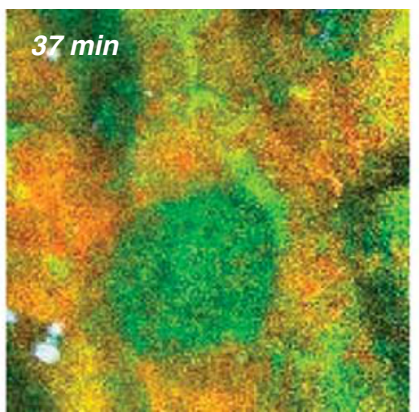

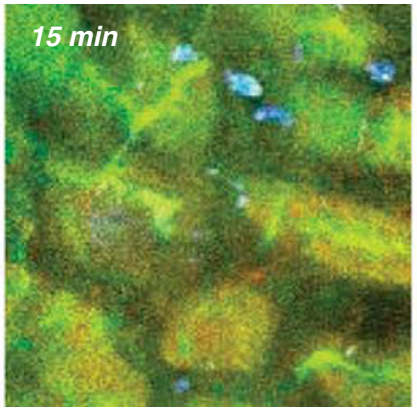

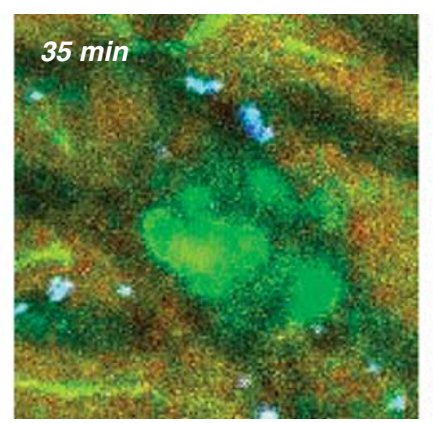
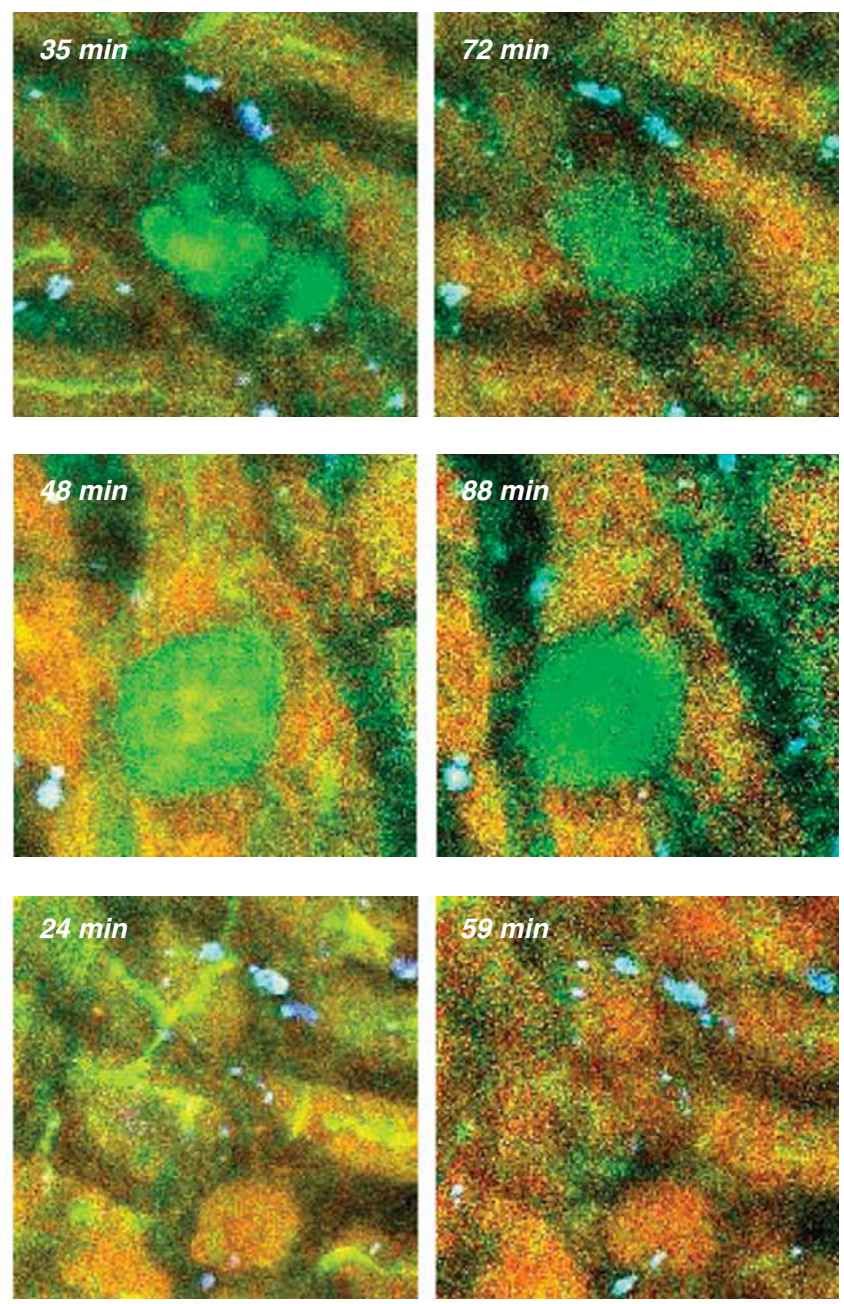

D

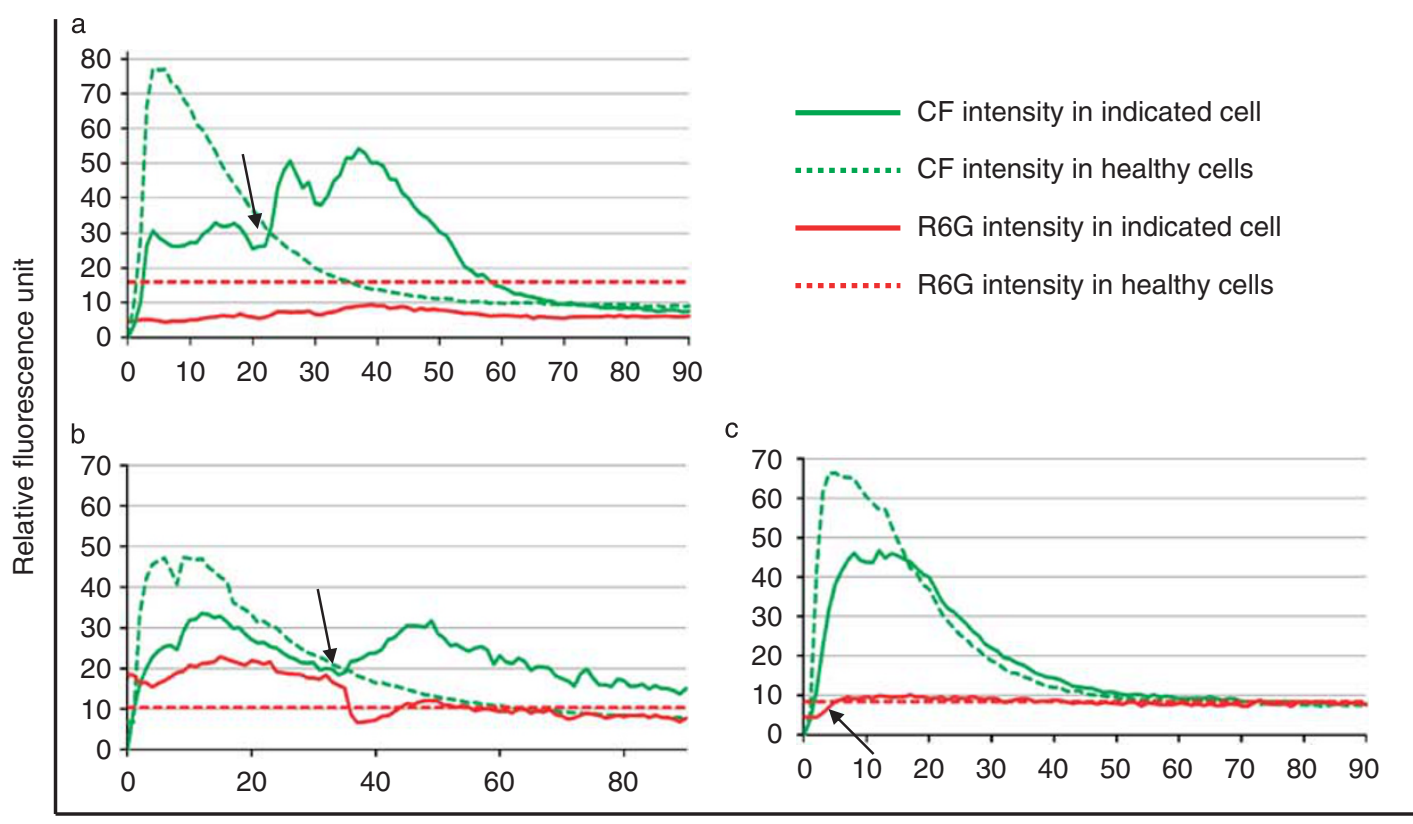

Time (min) post-CFDA injection 
torn canalicular membrane (Figure 7b). The remaining organelles became clustered (Figure 7c). The ground-glass appearance of the cytoplasm in this necrotic hepatocyte was postulated to be parallel to the spongy appearance described in scanning EM. ${ }^{16}$

Prevention of canalicular membrane rupture by $\mathbf{N}$ acetylcysteine. $\mathrm{N}$-acetylcysteine (NAC), a glutathione supplement and an antidote for APAP intoxication, was administered to determine whether it could reduce the occurrence of canalicular membrane rupture events. Figure 8 shows that administration of NAC significantly reduced the occurrence of NCFR cells (NAC $1.8 \pm 1.2$ versus control $6.4 \pm 3.5$ cells per microscopic area, $P<0.001$ ) but not ECFR cells (NAC $17.9 \pm 14.5$ versus control $20.4 \pm 18.0$ cells per microscopic area, $P=0.789$ ).

\section{Discussion}

Intravital imaging by multiphoton microscopy may give clues of crucial steps in biological events occurring inside the body. In this study, we recorded the steps of APAP-induced

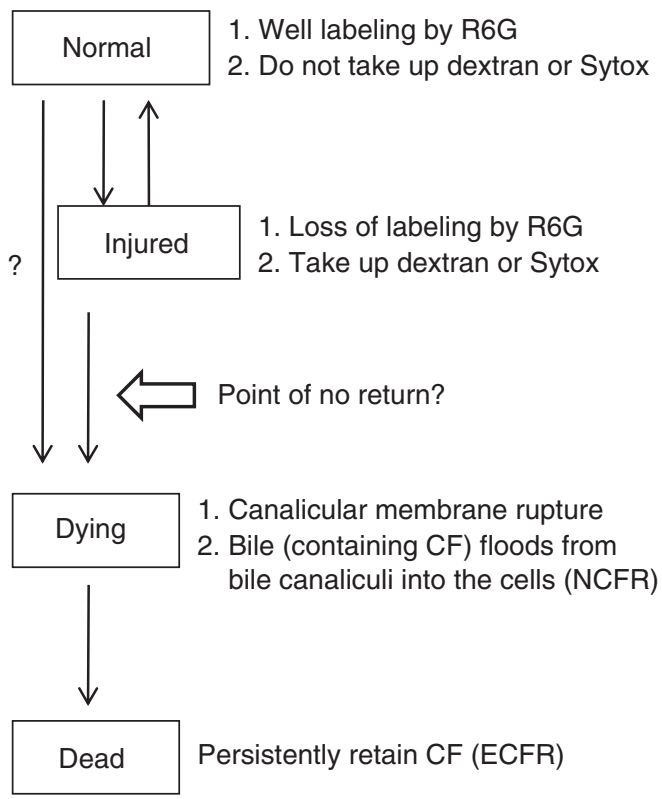

Figure 6 Schematic illustrations showing our hypothesis on the sequential morphological changes of APAP-induced hepatocyte injury. Normal hepatocytes became injured as manifested by loss of R6G labeling or uptake of either dextran or Sytox through On rupture of apical membrane, bile containing CF gushed from bile canaliculi into the damaged cells. Later on, bile persistently leaked into the cells as manifested by retaining CF. We proposed that rupture of canalicular membrane is a critical determinant of point of no return in the course of hepatocyte necrosis hepatocyte necrosis in mice using multiphoton microscopy with the aid of our established intravital liver imaging system. In the APAP-injured liver, we first found several hepatocytes that were labeled as abnormal by RITC-dextran and, among them, some retained CF (Figure 1a). These CF-retaining
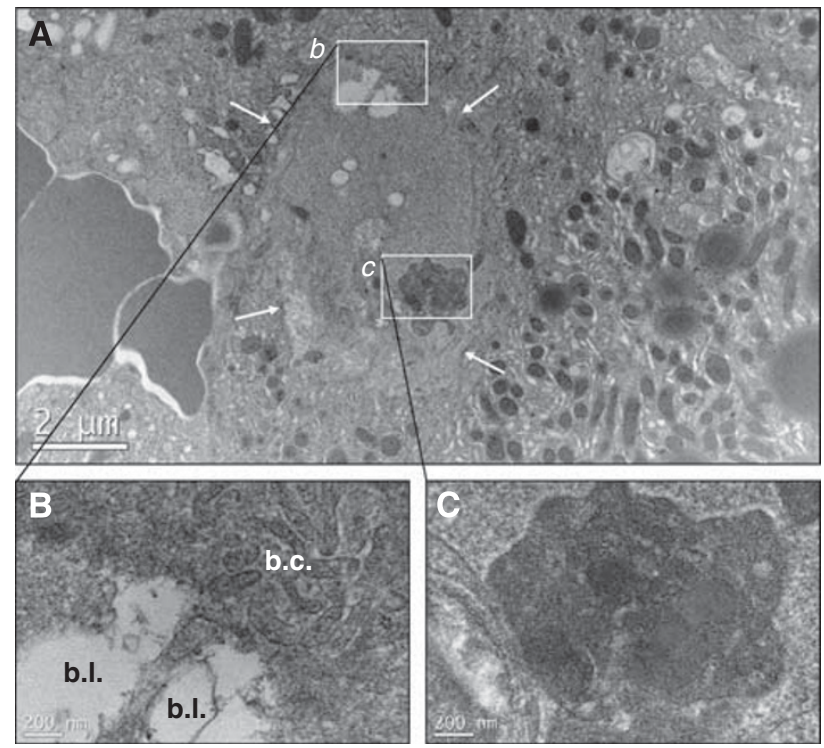

Figure 7 Transmission electron microscopy showing a dead hepatocyte able to depict consequences post-apical membrane rupture. (A) A shrunk hepatocyte indicated by arrows was devoid of organelles. Most of its content has ground-glass appearance. (B) Magnified photo of boxed area $b$ in $\mathbf{A}$ shows bile lakes with ragged margins. (C) Magnified photo of boxed area $c$ in $\mathbf{A}$ shows remaining organelles in the dead hepatocyte were clustered together. b.c. = bile canaliculus; b.l.=bile lakes. Scale bars indicate $2 \mu \mathrm{m}$ in $\mathbf{A}$ and $200 \mathrm{~nm}$ in $\mathbf{B}$ and $\mathbf{C}$
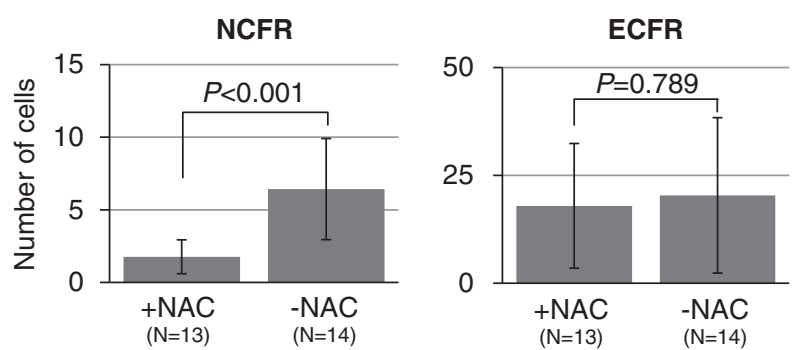

Figure 8 Prevention of canalicular membrane rupture in hepatocytes by NAC treatment in APAP-intoxicated mice. The numbers of NCFR and ECFR cells per $660 \times 660 \mu \mathrm{m}$ microscopic area were recorded in 13 NAC-treated ( + NAC) and 14 non-treated $(-N A C)$ mice. The mean number of NCFR cells in NAC-treated group was statistically significantly lower than NAC-non-treated group $(P<0.001)$ but the number of ECFR was not different in both groups $(P=0.789)$. Comparisons of the means were performed by Mann-Whitney U-test

Figure 5 Three kinds of APAP-injured cells based on the staining of the mitochondrial dye R6G. In addition to R6G, CFDA and Sytox were also injected. Cells indicated by arrows in $\mathbf{A}, \mathbf{B}$, and $\mathbf{C}$ were serially observed. Kinetics of $\mathrm{CF}$ and R6G in these cells were shown in $\mathbf{D}$. (A) A cell not labeled by R6G but stained by Sytox developed apical membrane rupture later. (B) A cell originally well labeled by R6G developed simultaneously bile flooding into cell and loss of R6G staining. (C) A Sytox-stained cell recovered its R6G labeling. (D) Kinetics of CF and R6G for these three cells in comparison with normal hepatocytes. Plot a, b, and c are for the indicated cells in A, B, and C, respectively. Note resurgence of $\mathrm{CF}$ fluorescence (arrows) in a and b, simultaneous drop of R6G fluorescence in b and re-elevation of R6G fluorescence (arrow) in c. Scale bars indicate $20 \mu \mathrm{m}$ 
hepatocytes were further separated into NCFR and ECFR cells, according to when they commenced CF retention during microscopic observation. Figures 2 and 3 show that NCFR cells were those with new canalicular membrane ruptures and backward flooding of bile from bile canaliculi into hepatocytes. Blebs protruded and dislodged into sinusoids might be frequently seen. Though it was speculated that the release of hepatocyte contents into circulation must be the source for serum aminotransferase elevation in patients with hepatitis, ${ }^{1}$ this is the first time, to our knowledge, proving it and particularly showing the release of hepatocellular contents is in the form of dislodged blebs. ECFR cells were those already ruptured at that membrane. Backward flooding of bile into hepatocytes after canalicular membrane rupture indicated the existence of a physiological or pathophysiological hydrostatic pressure gradient between bile canaliculi and hepatocytes. These violent events are impossible to observe in cultured cells because the cell culture system is unable to create such a pressure gradient. Several factors are known to increase individual susceptibility to APAP, such as increased CYP2E1 activity and lower baseline levels of glutathione. ${ }^{20}$ Here we hypothesize that elevation of bile duct pressure and hence increased hydrostatic pressure gradient between bile canaliculi and hepatocytes may also be a factor. This may explain the common elevation of serum aminotransferases in patients with obstructive jaundice ${ }^{21}$ and suggest elevation of bile duct pressure per se contributes to the induction of hepatocyte necrosis.

The labeling of hepatocytes by RITC-dextran indicated that sinusoidal membrane disruption occurred in these cells. In vitro studies on anoxia-induced cell death revealed that initial membrane disruption only allowed permeation to molecules of lower molecular weights. Progressive injury then allowed permeation to molecules of higher molecular weights. ${ }^{22,23}$ Physiologically, the plasma membrane indicated here represented the capillary-facing basal membrane. Preceding canalicular membrane ruptures, cells 4 and 5 in Figure 3, were permeable only to Sytox but not dextran, suggesting that their basal (sinusoidal) membrane defects might be minor when compared with the cell in Figure 2a. Nevertheless, they all experienced canalicular membrane rupture, suggesting that the severity of basal membrane disruption may not be the only determinant for the occurrence of canalicular membrane rupture. The fact that CF-retaining cells were usually pre-labeled by RITC-dextran (table of Figure 1d, and more clearly the cells in Figures $2 a$ and b) suggested that sinusoidal membrane disruption usually occurred before canalicular membrane rupture.

It would be interesting to know whether the APAP-injured hepatocytes could be reversed. The rarely seen cell in Figure $5 c$ clearly demonstrated the reversibility of mitochondrial membrane potential, which is a useful indicator of cell vitality. Hydrophobic bile acids in bile were toxic to cells through their detergent action and oxidation effects. ${ }^{24}$ Retention of these acids in cholestatic diseases would lead to hepatocyte death, not to mention the sudden flooding of bile into hepatocytes after canalicular membrane rupture. This may explain the cell in Figure $5 b$, which exhibited a sudden loss of mitochondrial membrane potential in parallel with canalicular membrane rupture (Figure 5Db). The ground- glass substance inside the dead hepatocyte shown in Figure 7 might be the result of the esterification effects of bile acids. This severe intracellular structural damage is likely irreversible.

We confirmed that glutathione supplemented by NAC administration reduced the occurrence of canalicular membrane rupture in APAP-induced liver injury (Figure 8). This further supported the fact that canalicular membrane rupture is a critical determinant in APAP-induced hepatocyte necrosis. The ECFR cells are already irreversibly dead cells and are not reduced by NAC administration. Thus, the initially injured hepatocytes at stages of basal membrane disruption or mitochondrial dysfunction (Figure 6) are the targets for NAC rescuing. NAC may stop the progression or even reverse the injury. The progression of injury in individual hepatocytes may be determined by their own glutathione pools remained. But how depleted the cellular glutathione as a threshold for irreversible injury is still unknown. Clinically, administration of NAC is dependent on serum APAP levels above the treatment line on the Rumack and Matthew ${ }^{25}$ nomogram. This measurement may reflect the total glutathione pool in the liver.

Currently, morphological criteria for defining cell necrosis are based on plasma membrane blebbing and rupture. ${ }^{1-4}$ Detailed information on the steps of cell necrosis in vivo remains unclear. Dozens of methods already exist to study cell death events; however, studies using intravital imaging are still lacking. ${ }^{5}$ This study on APAP-induced hepatocyte necrosis by intravital microscopic imaging disclosed a previously hidden but critical step of canalicular membrane rupture and the flooding of bile into cells. This event is possibly one of the determinants for the injured hepatocytes to cross beyond the point of no return in the process of hepatocyte injury (Figure 6). These critical phenomena have been overlooked because of inadequate accessibility to this knowledge when using conventional experimental techniques. We have shown the important role of intravital microscopic imaging techniques on studying cell biology issues such as cell death.

It remains to be shown whether the rupture of the apical membrane in cell death is a unique hepatocytes phenomenon or is a universal event for glandular epithelial cells. Nevertheless, our present results should make researchers focus on the integrity of the apical membrane, at least in hepatocytes, in studying cell death. Further fundamental research in this area may lead to the development of novel therapeutic strategies in treating hepatocyte death.

\section{Materials and Methods}

Animal experimental procedures. C57BL/6J mice, each weighing approximately $20 \mathrm{gm}$, were used. The experimental procedures, including installation of the liver window device on the mouse abdomen, were as previously described ${ }^{10}$ and were approved by the Institutional Animal Care and Use Committee, National Taiwan University College of Medicine. An overdose of APAP $(500 \mathrm{mg} / \mathrm{kg} \mathrm{BW}$, Sigma, St. Louis, MO, USA) was given through an intraperitoneal injection $24 \mathrm{~h}$ before microscopic examination. The dosages of intravenously injected fluorochromes before microscopic examination were: RITC or FITC conjugated with a large molecule dextran (70 000 daltons for RITC and 10000 daltons for FITC, Sigma), $10 \mathrm{mg} / \mathrm{mouse}$; CFDA (Sigma), $50 \mu \mathrm{g} / \mathrm{mouse}$; R6G (Sigma), $1 \mathrm{mg} / \mathrm{mouse}$; Sytox Blue (Molecular Probes, Eugene, OR, USA), $25 \mathrm{~nm} /$ mouse. NAC (China Chemical \& Pharmaceutical, Taipei, Taiwan) was intravenously administered at a single dose of $3 \mathrm{mg} /$ mouse, $30 \mathrm{~min}$ before microscopic examination. 
Multiphoton microscopy. The settings of the multiphoton microscope were as previously described. ${ }^{10}$ Fluorescence images were acquired serially on the same microscopic areas at 1-min intervals. Zero time was before CFDA but after RITCdextran injection. The fluorescence signals at $500-550 \mathrm{~nm}, 550-630 \mathrm{~nm}$, and $435-485 \mathrm{~nm}$ were recorded and then pseudocolored into green, red, and blue, respectively. Image J software (National Institute of Health, Bethesda, MD, USA) was used for image processing.

Analyzing fluorescence intensities of CF and R6G in hepatocytes. In the time series of images, areas in cells of interest were circled and the average fluorescence intensities (green channel for CF and red channel for R6G) per pixel of the areas circled were measured. The kinetic curves were then plotted.

Transmission electron microscopy. For TEM examination, the liver tissues were fixed with $5 \%$ glutaraldehyde in $0.2 \mathrm{M} / \mathrm{pH} 7.3$ phosphate buffer. Ultrathin sections of gray interference color were observed with a JEM-1400 electron microscope (JEOL Ltd., Tokyo, Japan).

Statistics. Cell numbers per $660 \times 660 \mu \mathrm{m}$ microscopic area in the liver of APAP-injured mice treated with or without NAC were expressed as mean \pm S.D. Comparisons of the means were performed by Mann-Whitney U-test. $P$-values $<0.05$ were considered significant.

\section{Conflict of Interest}

The authors declare no conflict of interest.

Acknowledgements. This work was supported by grants from the National Science Council of Taiwan (NSC 97-3112-B-002-036 and NSC 98-2112-M-002008-MY3) and from National Taiwan University (99R80846). We thank the Joint Center for Instruments and Researches, College of Bioresources and Agriculture, National Taiwan University, for performing electron microscopy.

\section{Authors contribution}

FCL conducted experiments, collected data, prepared microscopic images, and wrote the manuscript. CJL, SSW, TLS, and SYL helped and participated in the acquisition of microscopic images. LLC conducted acetaminophen intoxication experiments in mice and carried out electron microscopic examination. WL developed software for analyzing images. GTH critically revised the manuscript. CYD sponsored the project, established multiphoton microscopic facilities, and analyzed the data. HSL designed the experiments and wrote the manuscript.

1. Lee WM. Drug-induced hepatotoxicity. N Engl J Med 2003; 349: 474-485.

2. Kroemer G, Galluzzi L, Vandenabeele P, Abrams J, Alnemri ES, Baehrecke EH et al. Classification of cell death: recommendations of the Nomenclature Committee on Cell Death 2009. Cell Death Differ 2009; 16: 3-11.

3. Kon K, Kim JS, Jaeschke H, Lemasters JJ. Mitochondrial permeability transition in acetaminophen-induced necrosis and apoptosis of cultured mouse hepatocytes. Hepatology 2004; 40: 1170-1179.

4. Golstein P, Kroemer G. Cell death by necrosis: towards a molecular definition. Trends Biochem Sci 2007; 32: 37-43.

5. Galluzzi L, Aaronson SA, Abrams J, Alnemri ES, Andrews DW, Baehrecke EH et al. Guidelines for the use and interpretation of assays for monitoring cell death in higher eukaryotes. Cell Death Differ 2009; 16: 1093-1107.

6. Helmchen F, Denk W. Deep tissue two-photon microscopy. Nat Methods 2005; 2: 932-940.

7. Kienast $Y$, von Baumgarten L, Fuhrmann M, Klinkert WE, Goldbrunner R, Herms J et al. Real-time imaging reveals the single steps of brain metastasis formation. Nat Med 2010; 16: 116-122.

8. Miller MJ, Wei SH, Parker I, Cahalan MD. Two-photon imaging of lymphocyte motility and antigen response in intact lymph node. Science 2002; 296: 1869-1873.

9. Liu Y, Chen HC, Yang SM, Sun TL, Lo W, Chiou LL et al. Visualization of hepatobiliary excretory function by intravital multiphoton microscopy. J Biomed Opt 2007; 12: 014014.

10. Li FC, Liu Y, Huang GT, Chiou LL, Liang JH, Sun TL et al. In vivo dynamic metabolic imaging of obstructive cholestasis in mice. Am J Physiol-Gastroint Liver Physiol 2009; 296: G1091-G1097

11. Stravitz RT, Kramer DJ. Management of acute liver failure. Nat Rev Gastroenterol Hepatol 2009; 6: 542-553.

12. James LP, Mayeux PR, Hinson JA. Acetaminophen-induced hepatotoxicity. Drug Metab Dispos 2003; 31: 1499-1506.

13. Burcham PC, Harman AW. Mitochondrial dysfunction in paracetamol hepatotoxicity: in vitro studies in isolated mouse hepatocytes. Toxicol Lett 1990; 50: 37-48.

14. Martin FL, McLean AE. Adenosine triphosphate (ATP) levels in paracetamol-induced cell injury in the rat in vivo and in vitro. Toxicology 1995; 104: 91-97.

15. Walker RM, McElligott TF, Massey TE, Racz WJ. Ultrastructural effects of acetaminophen in isolated mouse hepatocytes. Exp Mol Pathol 1983; 39: 163-175.

16. Walker RM, Racz WJ, McElligott TF. Scanning electron microscopic examination of acetaminophen-induced hepatotoxicity and congestion in mice. Am J Pathol 1983; 113: 321-330.

17. Gear AR. Rhodamine $6 \mathrm{G}$. A potent inhibitor of mitochondrial oxidative phosphorylation. J Biol Chem 1974; 249: 3628-3637.

18. Baatz H, Steinbauer M, Harris AG, Krombach F. Kinetics of white blood cell staining by intravascular administration of rhodamine 6G. Int J Microcirc Clin Exp 1995; 15: $85-91$.

19. Elmore SP, Nishimura Y, Qian T, Herman B, Lemasters JJ. Discrimination of depolarized from polarized mitochondria by confocal fluorescence resonance energy transfer. Arch Biochem Biophys 2004; 422: 145-152.

20. Kaplowitz N. Acetaminophen hepatotoxicity: what do we know, what don't we know, and what do we do next? Hepatology 2004; 40: 23-26.

21. Hayat JO, Loew CJ, Asrress KN, McIntyre AS, Gorard DA. Contrasting liver function test patterns in obstructive jaundice due to biliary strictures and stones. QJM 2005; 98: 35-40.

22. Chen J, Liu X, Mandel LJ, Schnellmann RG. Progressive disruption of the plasma membrane during renal proximal tubule cellular injury. Toxicol Appl Pharmacol 2001; 171: $1-11$

23. Dong Z, Patel Y, Saikumar P, Weinberg JM, Venkatachalam MA. Development of porous defects in plasma membranes of adenosine triphosphate-depleted Madin-Darby canine kidney cells and its inhibition by glycine. Lab Invest 1998; 78: 657-668.

24. Perez MJ, Briz O. Bile-acid-induced cell injury and protection. World J Gastroenterol 2009; 15: $1677-1689$.

25. Rumack BH, Matthew H. Acetaminophen poisoning and toxicity. Pediatrics 1975; 55 : 871-876.

cc

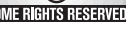
licensed under the Creative Commons Attribution-Noncommercial-Share Alike 3.0 Unported License. To view a copy of this license, visit http:// creativecommons.org/licenses/by-nc-sa/3.0/

Supplementary Information accompanies the paper on Cell Death and Disease website (http://www.nature.com/cddis) 